\title{
Análise de Sobrevivência na Modelagem do Tempo de Vida de Redes de Sensores sem Fio
}

\author{
Rodrigo Teles Hermeto $^{1}$, Juvêncio Santos Nobre ${ }^{2}$ e Danielo G. Gomes ${ }^{1}$ \\ ${ }^{1}$ Grupo de Redes de Computadores, Engenharia de Software e Sistemas (GREat) \\ ${ }^{2}$ Departamento de Estatística e Matemática Aplicada (DEMA) \\ Universidade Federal do Ceará (UFC) - Fortaleza, CE - Brasil \\ \{rodrigohermeto, dgomes\}@great.ufc.br, juvencio@ufc.br
}

\begin{abstract}
Resumo. As Redes de Sensores Sem Fio (RSSF) são exemplos de ResourceConstrained Networks (RCNs) nas quais recursos de processamento, armazenamento e energia são limitados. Estimar a estrutura probabilística do tempo de vida de uma RSSF antes da sua implantação permite, por exemplo, um melhor planejamento de manutenção preventivas. Este artigo aborda os modelos exponencial, Weibull e log-normal, comumente utilizados em estudos de Análise de Sobrevivência, para obter estimativas do tempo de vida de uma rede de sensores real a partir dos tempos de vida observados de seus nós em simulação. Aqui propomos resposta a uma questão em aberto na literatura: por quanto tempo a rede permanecerá em funcionamento.
\end{abstract}

\begin{abstract}
Wireless sensor networks are resource constrained networks whose processing capabilities, storage and energy are limited. Before its real/physical implantation, a maintenance planning can better estimate the network lifetime. This article discusses how the exponential, Weibull and log-normal models, commonly used in Survival Analysis studies, can better estimate the lifetime of a real sensor network. Here we propose to answer one question open in the literature: how long the network will remain in operation.
\end{abstract}

\section{Introdução}

Em um ambiente onde a energia é um recurso limitado, o tempo de vida é a métrica mais crítica. Assim é importante saber por quanto tempo uma determinada rede de sensores consegue monitorar o seu entorno, sobretudo quando se trata de um ambiente inóspito ou de difícil acesso. Entretanto, o entendimento do que vem a ser tempo de vida em redes de sensores não é consensual. Dietrich e Dressler (2009), por exemplo, consideram tempo de vida de uma RSSF na perspectiva de conectividade, cobertura e qualidade de serviço (QoS).

Independentemente da abordagem utilizada, notamos na literatura que o tempo de vida em RSSF têm sido usado somente de forma reativa, i.e. após a morte da rede. Entretanto, conhecer o seu tempo de vida a posteriori dificulta a realização de manutenções preventivas, como troca de baterias ou reposição de nós.

Le et al. (2010) modelaram o tempo de vida dos nós de uma rede de sensores sem fio utilizando a distribuição exponencial em diferentes formas de organização dos nós. Até o presente momento, esse é o trabalho encontrado na literatura que se apresenta 
mais correlato à nossa proposta. Entretanto, como será mostrado na seção de resultados, a distribuição exponencial não apresenta estimativas precisas quando comparada a outros modelos paramétricos. Além disso, o modelo proposto de cálculo da probabilidade sugere que a rede seja considerada viva enquanto existir pelo menos um nó vivo capaz de fazer a comunicação entre dois pontos distintos da rede (e.g. entre um nó sensor e o sorvedouro). Contudo, em redes nas quais uma grande quantidade de nós já estejam mortos, considerar a rede viva com somente alguns poucos pode levar a um monitoramento inadequado.

Neste trabalho, propomos a utilização de três métodos probabilísticos paramétricos, a saber exponencial, Weibull e log-normal, com objetivo de avaliar estatisticamente o tempo de vida de uma rede em ambiente simulado antes da sua implantação. De acordo com Colosimo e Giolo (2006), essas distribuições têm-se mostrado bastante adequadas ao se modelar "tempo de falha".

\section{Função de Sobrevivência e Cálculo do Tempo de Vida}

A função de sobrevivência $S(t)$ (ou função de confiabilidade) fornece a probabilidade de um nó estar vivo após o instante $t$. Seguindo a definição de tempo de vida de Cerpa et al. (2004), cuja rede é considerada viva baseando-se na porcentagem de nós conectados ao(s) sorvedouro(s), pode-se estimar o tempo de vida da rede das seguintes maneiras:

1) se os tempos de vida dos nós seguem a distribuição exponencial $(\lambda)$ :

$$
t_{\text {vida }}(k)=-\ln (k) \lambda
$$

2) se os tempos de vida dos nós seguem a distribuição de Weibull $(\lambda, \gamma)$ :

$$
t_{v i d a}(k)=-\ln (k)^{\frac{1}{\gamma}} \lambda
$$

3) se os tempos de vida dos nós seguem a distribuição log-normal $(\mu, \sigma)$ :

$$
t_{v i d a}(k)=e^{\left(\mu-\Phi^{-1}(k) \sigma\right)},
$$

em que $t_{v i d a}(k)$ representa o quantil de ordem $k$ da referida distribuição.

\section{Material e Métodos}

Os experimentos deste artigo foram realizados utilizando 25 nós MicaZ com sistema operacional Contiki. Como ferramenta de simulação, utilizamos o Cooja, um simulador com suporte a diversas plataformas de hardware, dentre elas o MicaZ.

\subsection{Estratégia de estudo - delineamento}

O delineamento ilustra a estratégia da solução proposta neste artigo, organizada conforme a Figura 1.

\subsubsection{Calibração do simulador}

Esse processo consiste em utilizar variáveis aleatórias modeladas a partir de uma rede piloto e replicá-las no simulador com o objetivo fidelizá-lo conforme as mesmas condições dessa rede. Neste artigo, replicamos a variação de carga inicial de pilhas. A variação 


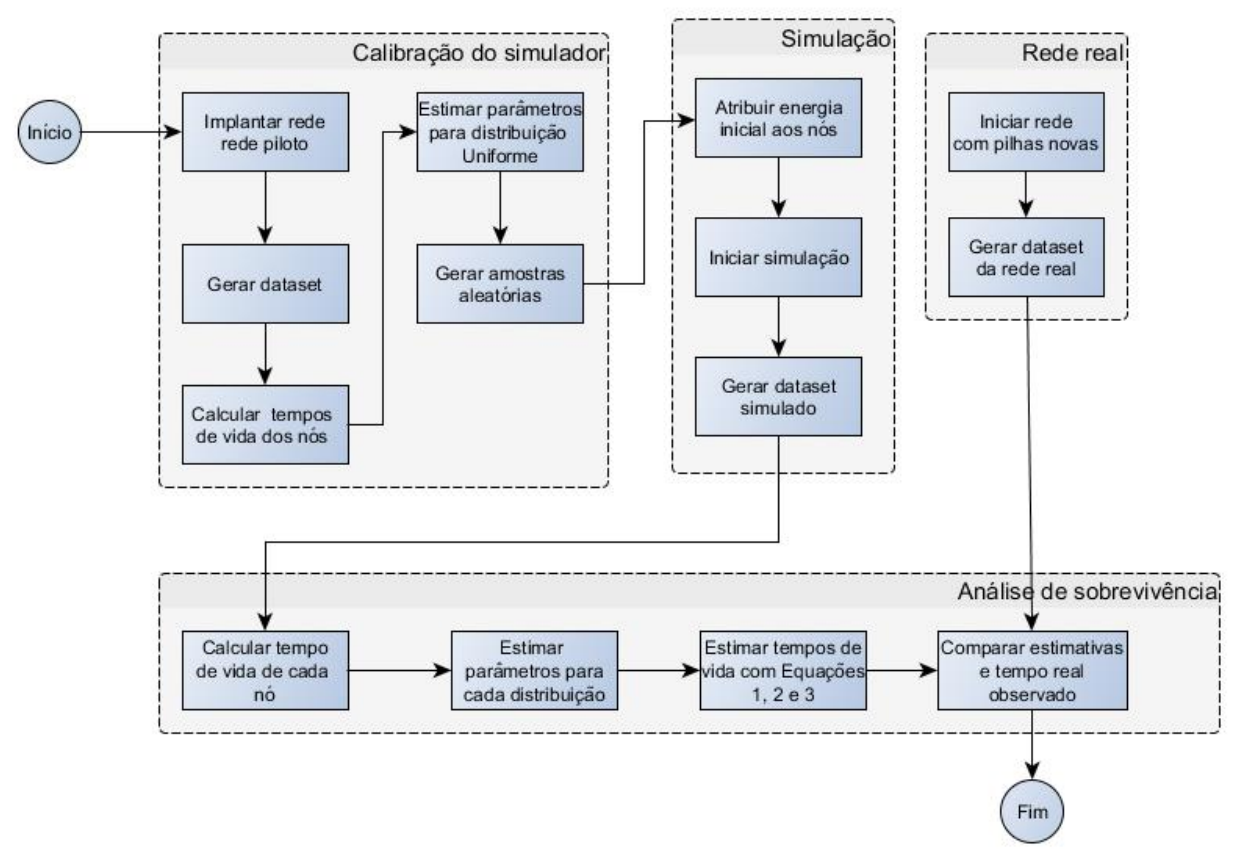

Figura 1. Delineamento.

Tabela 1. Tempos que indicam últimos registros de vida dos nós da rede piloto.

\begin{tabular}{|c|c|c|}
\hline Nó & Tempos 1 (h) & Tempos 2 (h) \\
\hline $\mathbf{1}$ & 106,2 & 106,4 \\
\hline $\mathbf{2}$ & 107,2 & 104,3 \\
\hline $\mathbf{3}$ & 103,1 & 109,7 \\
\hline $\mathbf{4}$ & 104,6 & 108,0 \\
\hline $\mathbf{5}$ & 108,6 & 103,7 \\
\hline $\mathbf{6}$ & 99,3 & 102,3 \\
\hline
\end{tabular}

aleatória da energia inicial em pilhas foi observada conforme Park et al. (2005). Os tempos de vida dos nós são apresentados na Tabela 1.

A carga energética inicial de uma pilha AA alcalina nova tem aproximadamente 9360 Joules [AllAboutBatteries 2011]. A plataforma MicaZ é alimentada por 2 pilhas, totalizando 18.720 Joules. Ao final da simulação, que durou aproximadamente dois dias, foi observado que os nós obtiveram tempo médio de vida de aproximadamente 94 horas. Sabendo que os nós vivem aproximadamente esse tempo no simulador, aplicamos o seguinte cálculo para estimar a energia inicial, em Joules, das pilhas do i-ésimo nó:

$$
\operatorname{Carga}_{\text {inicial }_{i}}=\frac{18720 \times T_{i}}{94}
$$

cujo $T_{i}$ representa o tempo de vida do i-ésimo nó da rede piloto.

Assumindo que a carga inicial é uniformemente distribuída, obtêm-se que as cargas iniciais das pilhas Duracell Alcalina AA foram geradas a partir da distribuição Uniforme contínua $(\min =19794, \max =21847)$. 


\subsubsection{Simulação e Rede Real}

Simulamos a rede mostrada na Figura 2 trinta vezes, gerando um dataset em cada simulação. Atribuímos valores aleatórios de energia inicial originados a partir da distribuição Uniforme contínua $(\min =19794$, $\max =21847)$. Em seguida, implantamos fisicamente a rede e essa foi deixada em execução enquanto o sorvedouro recebia pacotes oriundos dos nós sensores.

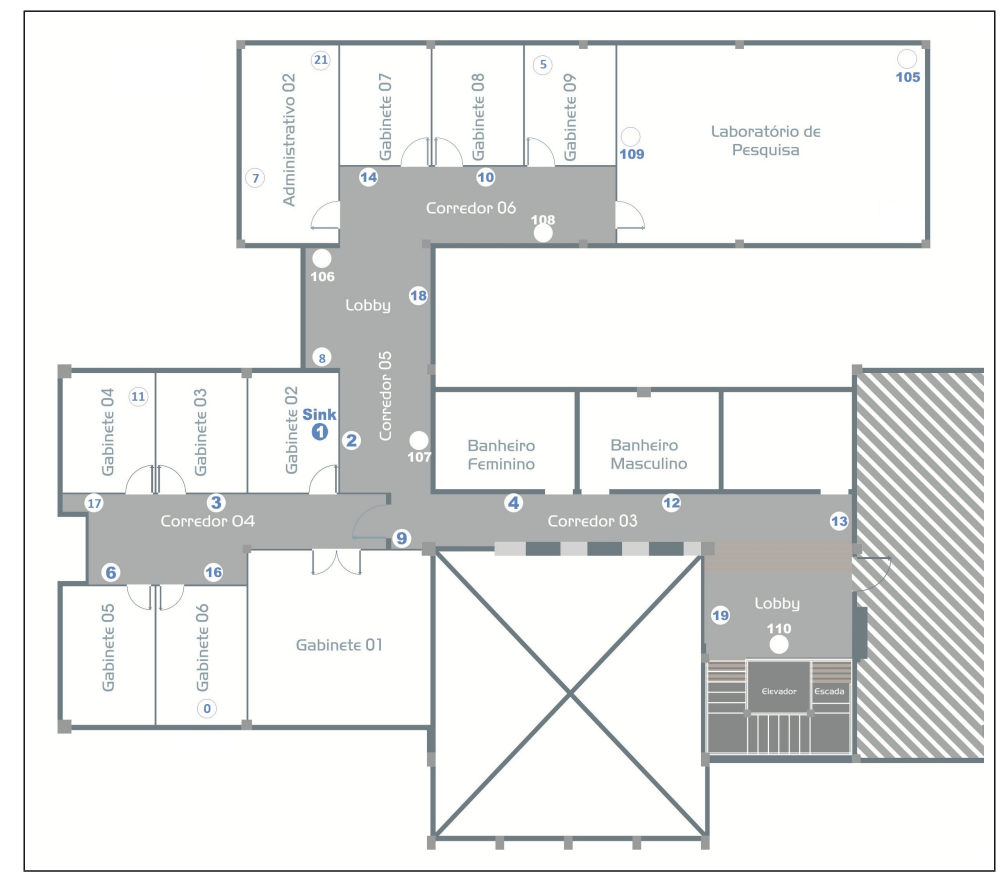

Figura 2. Rede utilizada nos experimentos.

\subsubsection{Análise de sobrevivência}

Ajustamos os tempos de vida dos nós observados nas simulações às distribuições exponencial, Weibull e log-normal, utilizando o estimador de máximo verossimilhança. Por fim, as Equações 1,2 e 3 foram utilizadas para estimar o tempo de vida da rede para cada modelo analisado.

\section{Resultados e discussões}

Ao final das 30 simulações e aplicando as Equação 1,2 e 3, obtivemos os resultados apresentados na Figura 3. Nela é possível observar as estimativas inferidas por cada modelo a partir dos datasets gerados em simulação e compará-las com o tempo observado na rede física, considerando $k=0.51$ e $95 \%$ de confiança.

Observa-se que o modelo exponencial (Figura 3a) não conseguiu acertar, em nenhum momento, o tempo real observado na rede física. O que é um indicativo que essa distribuição não é adequada para modelar tempo de vida de uma RSSF. Por outro lado, os modelos de Weibull (Figura 3b) e log-normal (Figura 3c) conseguiram se ajustar bem ao tempo observado na rede física. Dentre esses dois modelos, nota-se que o de Weibull tem um ajuste um pouco melhor que o log-normal, tornando esse modelo mais adequado para modelar o tempo de vida de uma RSSF, considerando o percentil analisado. 

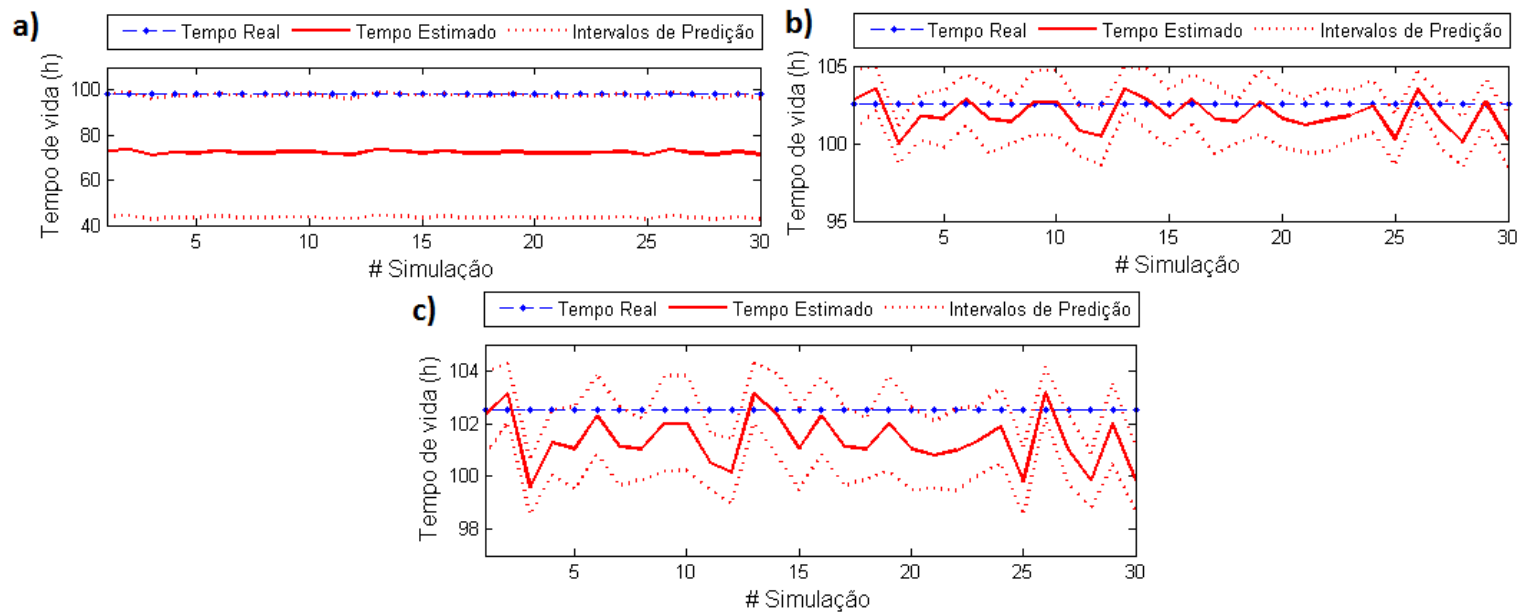

Figura 3. Estimativas obtidas a partir dos modelos: (a) exponencial, (b) Weibull e (c) log-normal.

\section{Conclusões e Trabalhos Futuros}

Neste trabalho, propomos a utilização da análise de sobrevivência em redes de sensores sem fio com objetivo de avaliar estatisticamente o desempenho de uma rede em ambiente simulado antes da sua implantação física e real. Para isso, avaliamos os modelos exponencial, Weibull e log-Normal na perspectiva de melhorar a estimativa do tempo de vida e o planejamento operacional de uma rede de sensores sem fio.

Como sequência deste trabalho, pretendemos diversificar os cenários dos experimentos, variando a quantidade de sorvedouros e a topologia da rede. Além disso, pretendemos testar outros modelos probabilísticos como, por exemplo, a distribuição de Birnbaum-Saunders.

\section{Referências}

AllAboutBatteries (2011). Find the energy contained in standard battery sizes. em: http://www.allaboutbatteries.com/energy-tables.html. acesso em: 15 dez. 2013.

Cerpa, A., Member, S., e Estrin, D. (2004). ASCENT: Adaptive Self-Configuring sEnsor Networks Topologies. 3(3):1-14.

Colosimo, E. e Giolo, S. (2006). Análise de sobrevivência aplicada. ABE - Projeto Fisher. Edgard Blücher.

Dietrich, I. e Dressler, F. (2009). On the lifetime of wireless sensor networks. ACM Transactions on Sensor Networks, 5(1):1-39.

Le, Z., Becker, E., Konstantinides, D. G., Ding, C., e Makedon, F. (2010). Modeling reliability for wireless sensor node coverage in assistive testbeds. Proceedings of the 3rd International Conference on PErvasive Technologies Related to Assistive Environments - PETRA '10, pp 1.

Park, C. e Lahiri, K. (2005). Battery discharge characteristics of wireless sensor nodes: An experimental analysis. In In Proceedings of the IEEE Conf. on Sensor and Ad-hoc Communications and Networks (SECON. 